\title{
Modernisation of the spectroscopic General Chapters in the United States Pharmacopeia (USP)
}

\section{Burgess ${ }^{a}$ and J.P. Hammond ${ }^{b}$}

aBurgess Analytical Consultancy Limited, 'Rose Rae', The Lendings, Startforth, Barnard Castle, Co. Durham, DL12 9AB, UK

bStarna Scientific Ltd, 52-54 Fowler Road, Hainault Business Park, Hainault, Essex, IG6 3UT, UK

As part of the USP-NF modernisation process, the General Chapters (Chemical Analysis) Expert Committee has and continues to review collections of chapters in a holistic approach in order to align concepts and content to ensure consistency. ${ }^{1}$ These revisions are intended to ensure that the scientific expectations defined in the chapters are aligned with current best practices to ensure suitability for use when the appropriate measurements are executed. To these ends, general chapters currently are grouped into two parts: $<1000$ chapters (i.e., those numbered below 1000), where the focus, as stated in the General Notices, is on content that may be required if called for in a monograph, and $>1000$ chapters (i.e., those numbered 10001999), where these chapters give information, definition or description.

The purpose of spectroscopic General Chapters is to provide the basis for establishing that an instrument or system is "suitable for use" in a USP monograph. This "suitability for use" is usually related to the technical performance specification of the instrument or system and represents a minimum standard. However, on its own, compliance to specification cannot provide overall "suitability for use" and best practice guidance provides the holistic component.
Hence "suitability for use" has two distinct aspects:

- Compliance with technical performance characteristics on the instrument/system itself,

- Operational best practices for an instrument/system when employed as part of a procedure stated in a monograph.

The instrument/system requirements currently described in General Chapter $\langle 851\rangle$, Spectrophotometry and Light Scattering, covered many spectroscopic techniques and had remained essentially unchanged over many years. Therefore, in the USP Review Cycle 2010 to 2015, it was decided to generate specific pairs of chapters for each of the main spectroscopic types. The below 1000 chapters would define minimum standards for compliance for use in a monograph, the paired above 1000 chapter would give theory, guidance and recommendations for best analytical practices. In general, below 1000 chapters include procedures, instrument qualification and validation/verification sections. Each section involves an assessment of method-specific requirements to ensure the suitability of the system and related measurements. General Chapter $\langle 851\rangle$, itself would be deleted after all the new chapters were approved.
However, it should be noted that this holistic approach has already been applied to two other spectroscopic chapters, currently in USP 37 2014, Nuclear Magnetic Resonance Spectroscopy $<761>$ and its paired best practices chapter Applications of Nuclear Magnetic Resonance Spectroscopy $<1761>$ and Mass Spectrometry $<736>$ and its paired best practices chapter Applications of Mass Spectrometry $\langle 1736\rangle$.

The objective was to articulate the basic elements required before and during the execution of a spectroscopic measurement to ensure that suitability for use has been confirmed. As an example, the validation/verification requirements for spectroscopic methods are specific for the procedures in the chapters.

Many of these concepts are not new to USP. The changes to the spectroscopy chapters are consistent with those defined in Chromatography $\langle 621\rangle$; that is, the user must demonstrate that the system is "suitable for use". It is important that before execution of a measurement the analyst should demonstrate method-specific suitability for the instrument to ensure the integrity of the measurement.

The proposed new below 1000 chapters have a common structure irrespective of the spectroscopic technique namely: 


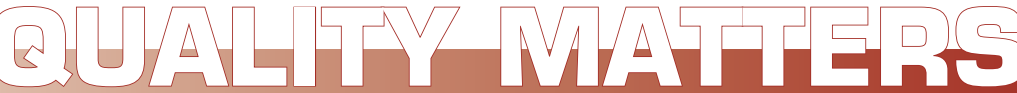

A short introduction to the particular technique and a link to the associated best practices chapter above 1000 ,

- Qualification requirements for IQ \& $\mathrm{OQ}$ with recommended procedures and the link to Analytical Instrument Qualification General Chapter $<1058>$,

- Specific critical attributes of analytical procedures involving the particular technique,

- Validation and verification needs as required by General Chapters $<1225\rangle$ and $<1226\rangle$ for alternative methods being developed for pharmacopeial monographs.

\section{Analytical instrument qualification (AIQ)}

Analytical Instrument Qualification $<1058>$ provides a risk-based framework for generating documented evidence over the lifecycle that the instrument or system is, and remains suitable for use in a monograph. $<1058\rangle$ is currently in revision with the intent of extending it to be compatible with GAMP $5^{2}$ and GAMP Laboratory Good Practice Guide $2^{\text {nd }}$ Edition. ${ }^{3}$ An article outlining proposals for change has been published. ${ }^{4}$ In addition, the extension proposal has been published. ${ }^{5}$

AIQ is currently defined as the collection of documented evidence that an instrument performs suitably for its intended purpose. Use of a qualified instrument in analyses contributes to confidence in the validity of generated data. Chapter $<1058>$ further characterises the steps in instrument qualification during AIQ:

- Design Qualification (DQ): The documented collection of activities that define the functional and operational specifications of the instrument and criteria for vendor selection based on the instrument's intended purpose.

- Installation Qualification (IQ): The documented collection of activities necessary to establish that an instrument is delivered as designed and specified, that it is properly installed in the selected environment and that this environment is suitable for the instrument.

- Operational Qualification (OQ): The documented collection of activities necessary to demonstrate that an instrument will function according to its operational specification in the selected environment. The extent of $\mathrm{OQ}$ testing that an instrument undergoes depends on its intended applications. Therefore, no specific $\mathrm{OQ}$ tests for any instrument or application are offered in $<1058>$. It should be noted that routine analytical tests do not constitute $\mathrm{OQ}$ testing.

- Performance Qualification (PQ): The documented collection of activities necessary to demonstrate that an instrument consistently performs according to the specifications defined by the user and is appropriate for the intended use. $P Q$ tests may resemble those performed during $\mathrm{OQ}$, but the specifications for their results may be set differently if required. Procedures for $\mathrm{OQ}$ and $\mathrm{PQ}$ testing that comply with regulatory expectations are not well defined and are subject to different interpretations.

The qualification tests and acceptance criteria are included in the below 1000 chapters to establish minimum pharmacopeial requirements for instrument performance. Ultimately, the use of a qualified instrument contributes to confidence in the validity of analytical data used to guarantee patient safety. The chapters will stipulate that manufacturerrecommended $\mathrm{OQ}$ and $\mathrm{PQ}$ tests and acceptance criteria may be used if deemed scientifically equivalent. This will prevent the AIQ tests in the $<1000$ chapters from becoming excessively prescriptive.

\section{Validation and verification}

As part of the overall revision process for spectroscopy general chapters, validation requirements also are being aligned with current standards. The validation of spectroscopic procedures

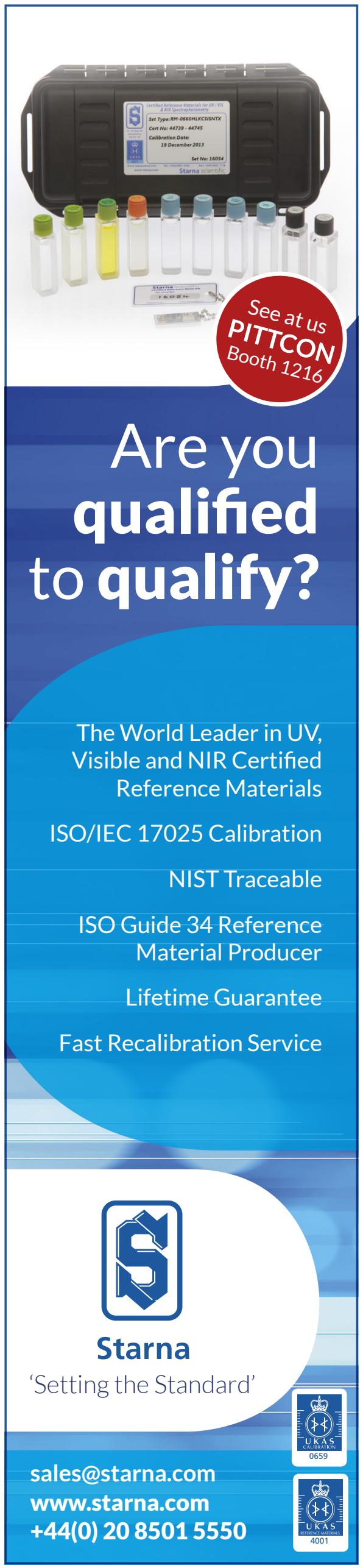

SPECTROSCOPYEUROPE 27 


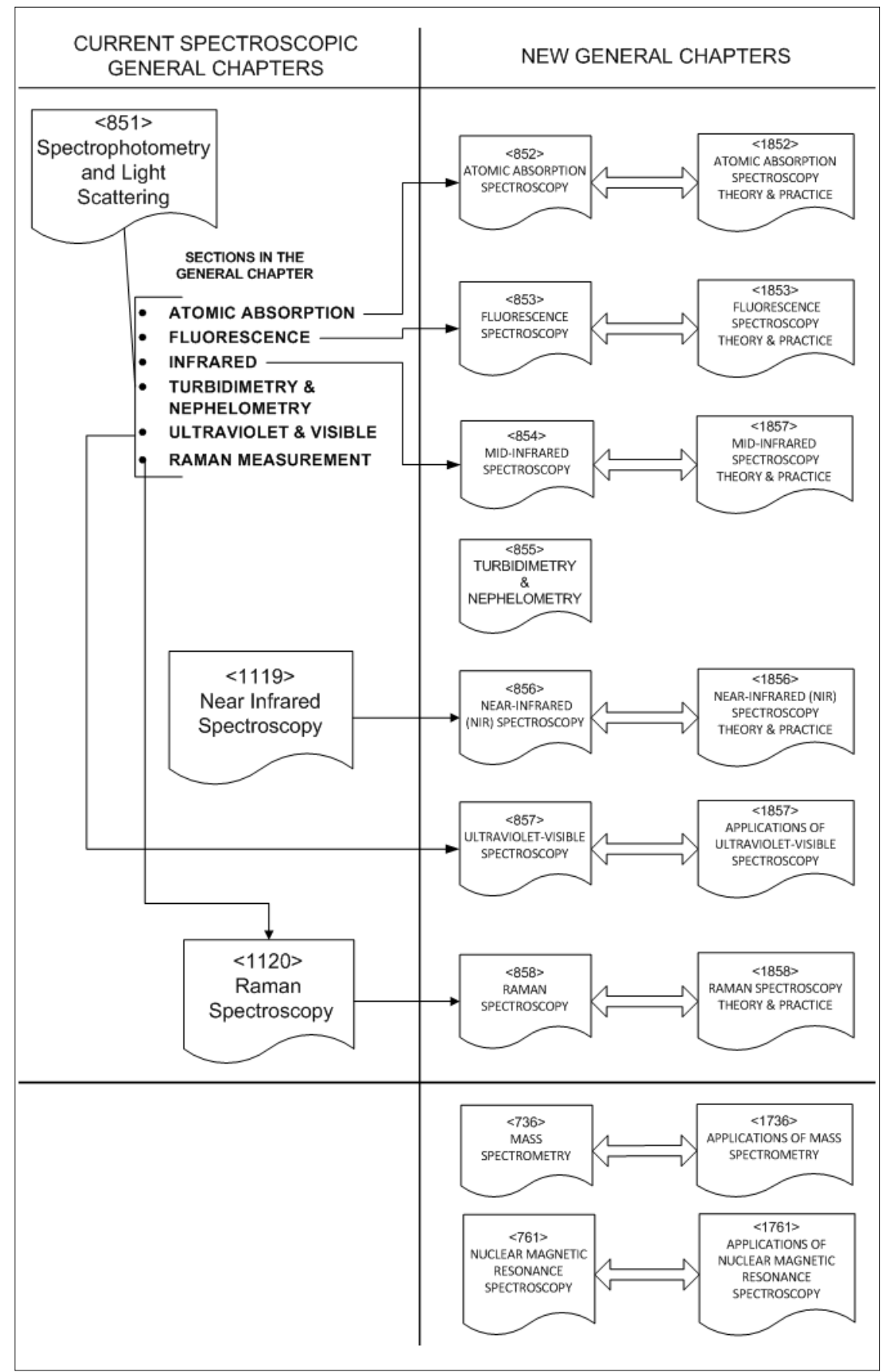

Figure 1. Revision Paths for Spectroscopic General Chapters.

is covered under both the ICH guidelines and Validation of Compendial Procedures $<1225>$ even though the predominant theme for each of these documents historically has been validation of chromatographic procedures. The objective of the current modernisation effort is to include validation sections in the various spectroscopy chapters thus providing both general direction for the validation of procedures based on these methods and specific validation requirements that are important for specific spectroscopic approaches.

It should be noted that all three general chapters related to validation and verification, $\langle 1224\rangle,\langle 1225\rangle$ and $<1226\rangle$, are currently under review and a Stimuli article has been published. ${ }^{6}$

\section{Revision of General \\ Chapter <851>}

As noted earlier, the current General Chapter <851> covers multiple spectroscopic types, and has not been subject to a major revision for many years. Whilst the general spectroscopic principles detailed in the current $<851>$ describe the fundamental requirements, it was felt that they are too vague to meet the extensive and increasing use of instrumental spectroscopic techniques, and the associated current (and future) regulatory requirements. In addition the proliferation of the application of new spectroscopic techniques makes revision of the current $<851>$ essential.

The following new spectroscopic chapter pairs have been developed in accordance with the approaches outlined previously:

- Atomic Absorption Spectroscopy, $<852>$ \& < 1852>

- Fluorescence Spectroscopy, $<853>$ $\&<1853>$

- Mid-Infrared Spectroscopy, $<854>\&$ $<1854>$

- Turbidimetry and Nephelometry, $<855>$

- Near Infrared (NIR) Spectroscopy, $<856>$ and $<1856>$

- Ultraviolet-Visible Spectroscopy, $<857>$ \& < 1857>

- Raman Spectroscopy, <858> \& $<1858>$

These together with other spectroscopic chapters are shown in relationship to the current $<851>$ in Figure 1, "Revision Paths for Spectroscopy General Chapters". Note that neither NIR nor Raman spectroscopies are adequately covered in <851> and had previously only above 1000 information chapters. This situation has now been rationalised.

Revision history during the 2010-2015 cycle

The drafts and revisions for these Spectroscopy General Chapters were 
published in Pharmacoeial Forum (PF) from 2011 to 2015. This is available from the USP website and is free. Back issues are also available. The USP standards are public standards and interested parties are requested to comment. We encourage everyone to subscribe and give input to this important process. All comments are made available to the Expert Committee for consideration and review. The journey to a final new or revised standard is carefully procedurally controlled and has to gain approval of the Council of Experts before it is made official. A synopsis of this process is given below.

- After extensive drafting and review at the start of the cycle, the six initial chapters: Atomic Absorption Spectroscopy, <852> and <1852>, Mid-Infrared Spectroscopy, <854> and $\langle 1854\rangle$ and Ultraviolet-Visible Spectroscopy, <857> and <1857> were published in late 2011, in PF 37(5).

- Following the comment period, the only chapter which did not require substantial revision was $<1857>$, the Ultraviolet-Visible Spectroscopy general information chapter.

- In early 2013, the two new Fluorescence chapters, namely $<853>$ and $<1853>$ were published in $P F$ 39(1). The five, reviewed and revised, remaining chapters from the initial publication set were again submitted for comment in PF 39(2). The "remains" of <851> was renamed "Light Scattering" and published in PF 39(3).

- Once again, significant comments were received and reviewed and in early 2014, these five chapters, i.e. $\langle 852\rangle$, <854>, <857>, <1852> and $\langle 1854\rangle$, together with the reviewed Fluorescence Spectroscopy, $<853>$ \& < 1853>, were published in PF 40(1).

- Having now disseminated <851> into the appropriate technique specific chapters, some techniques were not covered. Nephelometry, Turbidimetry and Visual Comparison were assigned to a new <855> chapter, which was published at the end of 2014, in PF 40(6).

- Also in PF 40(6) are the rationalised and revised Raman chapters <858> and $\langle 1858\rangle$, derived from the existing $<1120>$ which were open for comment until 31 January 2015.

- In PF 41 (1) 2015 the rationalised and revised NIR chapters <856> and $\langle 1856\rangle$, derived from the existing $\langle 1119\rangle$, were published and open for comment until 31 March 2015.

- Therefore, the following chapters become official in USP 38 (1 May 2015):

$\square$ Atomic Absorption Spectroscopy, $<852>$ \& < 1852>

$\square$ Fluorescence Spectroscopy, $<853>$ \& < 1853>

$\square$ Mid-Infrared Spectroscopy, $<854>$ \& <1854>

$\square$ Ultraviolet-Visible Spectroscopy, $<857>$ \& < 1857>

- However, monographs will still reference $\langle 851\rangle$. In other words the chapters will be official but their use is not mandatory because they are not referenced in any monograph. This will provide one year of transition.

In USP 39 (1 May 2016) all the references to $<851>$ will be eliminated and replaced by the appropriate new chapters. This is when the chapters became fully applicable.

\section{The future [2015-2020 cycle]}

In addition to the publication of the revised NIR and Raman chapters, the associated supportive science of Chemometrics is undergoing an extensive review, as are the fundamental concepts of Validation and Verification.

So again, the next cycle is going to be an exciting challenge. We do not yet know who will be involved in the next cycle, because the new Council of Experts will not be elected until April 2015 and the resultant Expert Committees will be appointed shortly after that. The new cycle begins officially in July 2015.
From a spectroscopic viewpoint, the new Chemometrics Chapter will be very important. In addition CD/ORD, XRF and XRD may be included in the cycle. At present there are no definite plans for a companion chapter $<1855>$ for <855> Nephelometry, Turbidimetry and Visual Comparison, although it may well happen especially as there is growing interest in these techniques from a bioanalytical perspective. In addition there is likely to be a revision of Analytical Instrument Qualification General Chapter $<1058>$

We hope that practicing spectroscopists will make the time and effort to keep in touch with these developments and comment. Over the years we have heard many practitioners complaining about the appropriateness of monographs and General Chapters. You now have the chance to assist with the change process. We strongly encourage you to use it!

\section{References}

1. "An alignment of concepts and content across the spectroscopy general chapters in the United States Pharmacopeia-National Formulary", Pharmacopeial Forum 40(1) (Jan.-Feb. 2014).

2. GAMP $P^{\circ}$ 5: Risk-Based Approach to Compliant GXP Computerized Systems. International Society for Pharmaceutical Engineering (ISPE), 5th Edition (February 2008). http://bit.ly/16ydrlt

3. GAMP ${ }^{\circ}$ Good Practice Guide: Risk-Based Approach to GxP Compliant Laboratory Computerized Systems. International Society for Pharmaceutical Engineering (ISPE), 2nd Edition (October 2012)

4. C. Burgess and R.D. McDowall, "Focus on quality; an integrated risk assessment for analytical instruments and computerized systems", Spectroscopy 28(11), 20-27 (2013).

5. L. Vuolo-Schuessler, M.E. Newton, P. Smith, C. Burgess and R.D. McDowall, "Harmonizing USP <1058> and GAMP for analytical instrument qualification", Pharmaceutical Engineering 34(1), 1-8 (2014). http:// www.ispe.org/pharmaceutical_engineering/ january2014

6. "Lifecycle management of analytical procedures: method development, procedure performance qualification, and procedure performance verification", Pharmacopoeial Forum 39(5) (2013). http://www.usp. org/usp-nf/notices/stimuli-article-lifecyclemanagement-analytical-procedures-postedcomment 